Instituto Internacional de Investigación y Desarrollo Tecnológico Educativo INDTEC, C.A.

DOI: https://doi.org/10.29394/scientific.issn.2542-2987.2016.1.2.12.200-217

OAI-PMH: http://www.indteca.com/ojs/index.php/Revista Scientific/oai

\title{
El Gerente del Siglo XXI ante los Retos del Pensamiento Estratégico
}

\author{
Autora: María Mercedes Carrillo \\ Universidad Nacional Experimental "Rafael María Baralt", UNERMB \\ mercecar-42-68@hotmail.com \\ Trujillo, Venezuela
}

\section{Resumen}

El gerente del siglo XXI ante los retos del pensamiento estratégico, observa que las organizaciones están frente a la exploración de desafíos futuros tanto previsibles como imprevisibles, más que preparar a las mismas para un posible mañana único. De allí, que la investigación que se desarrolla constituye un artículo cuyo objetivo es reflexionar sobre la gerencia del siglo XXI basada en el pensamiento estratégico; en este sentido, se tiene que la metodología usada para el estudio fue de tipo descriptiva y documental; los resultados evidencian claramente el perfil del gerente del siglo XXI como un guía orientador, creativo, innovador, estratega, llegando a concluir que: la dinámica profesional de las organizaciones manifiestan comportamientos según el cual las que mejor se adapten al entorno serán las que perdurarán en el tiempo.

Palabras clave: gerencia; perfil del gerente; pensamiento estratégico. 


\section{The Manager of the Century to the Challenges Strategic Thought}

\section{Abstract}

The manager of the XXI century to the challenges of strategic thinking, observes that organizations are facing future challenges exploring both predictable and unpredictable, rather than prepare them for a possible single morning. Hence, the research carried out is an article that aims to reflect on the management of the XXI century based on strategic thinking; in this sense, it is that the methodology used for the study was descriptive and documentary; the results clearly show the profile of the manager of the XXI century as a guiding guide, creative, innovative, strategist, reaching the conclusion that: professional dynamics of organizations manifest behaviors according to which best suited the environment will be those that will endure time.

Keywords: management; manager profile; strategic thinking. 


\section{Introducción}

El ambiente competitivo está cambiando a un ritmo muy acelerado. Las empresas que hasta hace solo unos años eran inmunes a la competencia global se miden hoy, en crueles batallas profesionales con rivales de todo el planeta. A nivel agrupado, algunas nuevas iniciativas estratégicas se han convertido en tareas esenciales para desarrollar las ventajas competidoras. Simultáneamente, se reconsideran conceptos claves del pensamiento estratégico y formas emergentes destinadas a explotar nuevos acontecimientos. Por ende, la tarea esencial de la Gerencia es llevar a cabo actividades con la participación de las personas, en la industria, comercio, organizaciones de servicios públicos, universidades, hospitales, instituciones militares o en cualquier forma de empresa humana.

La eficiencia con la que las personas trabajan en conjunto para el logro de un objetivo en común depende primordialmente de la capacidad de quienes dirijan la empresa, el avance tecnológico y el desarrollo del conocimiento no constituyen por sí solos elementos claves para el éxito, si no se combina con una acertada gerencia que combine su aplicación con los materiales y el talento humano del que se dispone. Es aquí donde a juicio de Añez, (2005) "cobra importancia la actividad de la gerencia, surgiendo la planificación, organización, dirección a seguir y la evaluación permanente de los procesos para verificar el grado de avance y la retroalimentación necesaria para consolidar, redimensionar y ajustar" (pág. 78).

Ahora bien, el problema actual está en cómo hacer para gerenciar en un contexto complejo y exigente, esto impone desafíos donde debe prevalecer una óptima cultura de gestión basada en la optimización de la capacidad de las personas, en todos los niveles operativos de la organización; el pensamiento estratégico está orientado a dar al gerente, los instrumentos conceptuales, las herramientas prácticas para la acción, y verificar lo que está sucediendo en su entorno, procurando establecer enfoques que faciliten la 
comprensión de lo nuevo, la necesidad de cambio hacia lo mejor, de ahí que el presente artículo aborda el tema del gerente del siglo XXI ante los retos que supone el pensamiento estratégico como factor clave en el desempeño organizacional.

Asimismo, las tareas de crear, implantar y ejecutar las estrategias de la organización constituyen el corazón y el alma de la administración de cualquier organización del mercado. La estrategia de una empresa es el "plan de acción" que tiene la gerencia para posicionar la empresa en la arena de su mercado, conducir sus operaciones, competir con éxito, atraer y satisfacer los clientes, como herramienta de idoneidad.

En este sentido, el éxito que puede tener la organización al alcanzar sus objetivos y también al satisfacer sus obligaciones sociales depende en gran medida, del desempeño gerencial de la organización. Esta afirmación, de que la gerencia es responsable del éxito o no de una empresa nos indica, por qué es necesaria la gerencia sin embargo, no nos muestra cuando ella es requerida, y la exigencia sucede siempre que haya un grupo de individuos con objetivos determinados.

Entretanto, las personas que asumen el desempeño gerencial de una organización se llaman "gerentes", y son los responsables de dirigir las actividades que ayudan a las organizaciones para alcanzar sus metas. Esta situación evidencia que, la base fundamental de un buen gerente abocado a las exigencias del siglo XXI, es la medida del poder y la actividad que éste tenga para lograr las metas de la organización. Es saber reducir al mínimo los recursos usados, para alcanzar los objetivos de la organización (hacer las cosas bien) y la capacidad para determinar los objetivos apropiados (hacer lo que se debe hacer).

Cabe decir, que ningún grado de eficiencia puede compensar la falta de eficacia, de manera tal que, un gerente ineficaz no puede alcanzar las metas de la organización, la eficacia es la clave del éxito de las organizaciones. Pero 
el ser gerente no sólo es dirigir actividades, es saber el proceso de cómo penetrar en esas acciones que realizan los miembros del grupo con el cual se trabaja. El gerente para poder lograr sus objetivos debe saber cómo usar las diferentes formas del poder para influir en la conducta de sus seguidores en distintas formas, sin olvidar que es lo que se quiere lograr y hacia dónde va.

De esta manera, la temática en cuestión presenta la gestión del gerente del siglo XXI, como el cargo que ocupa el director de una empresa, lo cual tiene dentro de sus múltiples funciones, representar a la sociedad frente a terceros y coordinar todos los recursos a través de los procesos gerenciales cuyo propósito no es más que lograr los objetivos establecidos. Al respecto, los postulados de Sisk y Sverdlik (1979) establecen que:

... El término gerencia es difícil de definir, significa cosas diferentes para personas diferentes. Algunos lo identifican con funciones realizadas por empresarios, gerentes o supervisores, otros lo refieren a un grupo particular de personas. Para los trabajadores; gerencia es sinónimo del ejercicio de autoridad sobre sus vidas de trabajo... (pág. 102).

En otras palabras, esta acepción dada por los autores deja al libre matiz de cada persona, de qué manera puede ser definida la acción gerencial, el gerente en su "deber ser", es el garante de la organización, sus decisiones deben estar dirigidas a buscar las soluciones pertinentes sin dañar a terceros y que sean favorables para el contexto, personal de la institución a su cargo. No obstante, en muchos casos la gerencia cumple numerosas funciones porque la persona que desempeña el rol de gerenciar tiene que desenvolverse como administrador, supervisor, delegado, etc. De allí, el problema de establecer una definición concreta de ese término.

Ante la situación planteada, el objetivo general de la indagación se presenta como analizar características del gerente del siglo XXI, ante los retos del pensamiento estratégico, entendiendo que el gerente del siglo XXI debe seguir un patrón de criterios, una filosofía clara de la administración justa 
concepción de las personas, con una ideología que le permita ganar apoyo efectivo y colaboradores comprometidos con su gestión, teniendo el gerente la capacidad de administrar, cambiar la visión simplista de su gestión por una permanente, compleja, pensar en infinitos caminos.

Por otra parte, el gerente del siglo XXI debe manejar todas las herramientas tecnológicas al alcance de sus manos asumiendo la capacidad de enseñar a sus colaboradores para formar un equipo de trabajo. Además, los gerentes del siglo XXI han cambiado mucho respecto a épocas anteriores. Hoy, lejos han quedado el autoritarismo y la individualidad para dar paso a conceptos como la creatividad del grupo, la comunicación o el liderazgo.

De los anteriores planteamientos se deduce que, el propósito del artículo en mención es reflexionar sobre la gerencia del siglo XXI basada en el pensamiento estratégico, todo ello, en base a un estudio de tipo descriptivo, documental, cualitativo. Hoy la gerencia plantea retos que debe asumir y actuar adecuadamente a favor de la sociedad específicamente en el entorno que le circunda su gestión.

En vista de los cambios, los nuevos esquemas gerenciales son reflejo de la forma como la organización piensa y opera, exigiendo entre otros aspectos: un trabajador con el conocimiento para desarrollar y alcanzar los objetivos del negocio; un proceso flexible ante los cambios introducidos por la organización; una estructura plana, ágil, reducida a la mínima expresión que crea un ambiente de trabajo que satisfaga a quienes participen en la ejecución de los objetivos organizacionales; un sistema de recompensa basado en la efectividad del proceso donde se comparte el éxito y el riesgo; y un equipo de trabajo participativo en las acciones de la organización.

Con respecto, a la gerencia basada en el Pensamiento Estratégico, las organizaciones en su afán de búsqueda de estrategias y herramientas innovadoras, originales, creativas han optado por seguir lineamientos basados en el pensamiento estratégico, porque éste es útil para elaborar planes o 
programas diseñados para alcanzar metas futuras y usar los recursos disponibles. El desarrollo de una estrategia permite diseñar enfoques, que ayudan a hacer frente a los retos planteados por el futuro.

Por esto, hay que definir la misión y las metas, analizando las ventajas, desventajas de cada línea de actuación, evaluando cada opción en términos de estrategia. Asimismo, en aras de seguir un único hilo conductor, es importante detenernos en el hecho de que en última instancia la gerencia estratégica es un modelo de cambio. No se trata de movernos si no, hacia dónde nos movemos. Aquí es el pensamiento estratégico el que juega un papel primordial. Debemos anticiparnos, prever el futuro, para conquistarlo.

En este orden de ideas, Ohmahe, (2004) afirma que el "pensador estratégico enfrenta problemas, tendencias y situaciones que parecen constituir un todo armonioso" (pág. 13). O lo que es lo mismo, el estratega debe desmembrar ese todo en sus partes constitutivas $y$, una vez que conoce el significado de cada parte, debe volver a juntarla aprovechando al máximo la ventaja competitiva de la empresa. La solución obtenida de esta forma, es distinta de la conseguida con el pensamiento lineal, ya que se ha identificado y estudiado los elementos concernientes a los problemas actuales y se han organizado de una manera relevante.

En ese sentido, se tiene que para el presente trabajo investigativo se considera como pensamiento estratégico la capacidad de poder crear un futuro mejor para cualquier organización por medio de la conjunción del análisis del entorno donde opera la empresa, a través del juicio razonado con las diferentes teorías gerenciales, con la aplicación del pensamiento creativo de los encargados de planificar los destinos organizacionales, para romper con lo convencional y diseñar estrategias, productos, servicios con ventajas competitivas superiores al de la competencia.

Sobre la base de las consideraciones anteriores, es importante la temática tratada en el artículo porque se presentan como bases del 
pensamiento estratégico la coordinación de varias mentes creativas dentro de una perspectiva común que le permita a un negocio avanzar hacia el futuro de una manera satisfactoria para todos. Esto es, para que se tengan herramientas como la de ayudar a explorar los muchos desafíos futuros, tanto previsibles como imprevisibles, más que prepararlo para un posible mañana único.

\section{Nueva Perspectiva: EI Gerente del Siglo XXI}

La ventana que abre nuevas perspectivas a las organizaciones actuales, amerita de gerentes fortalecedores de los canales participativos para la intervención activa de los trabajadores, al momento que identifican prioridades y formulan objetivos, además de ejercer un adecuado control sobre la organización. Al respecto, Añez, (ob. cit) establece el gerente "debe ser eficaz, promover la concertación interna, externa, haciendo compatibles los intereses en los distintos sectores $u$ organizaciones afines, coordinar esfuerzos y recursos para el logro de los objetivos económicos y sociales de la empresa" (pág. 80).

En otras palabras, en el gerente debe ser trascendental su actuación, poseer inteligencia para alcanzar los objetivos propuestos, identificar, evaluar oportunidades, amenazas, provenientes del contexto en que está inmerso a la vez que las analiza, para la consecución de cambios basados en conocimientos de actualidad. Ante esta realidad, las transformaciones dentro de la organización serán lideradas por el gerente quien habrá de estar formado, capacitado y adiestrado debido a la alta responsabilidad asignada a su persona para enfrentar con victoria los nuevos modelos relacionados con los procesos gerenciales de la nueva cultura colectiva.

Ante las nuevas realidades en las organizaciones, según Hernández, (2002), "se ha de formar, capacitar y perfeccionar al personal orientados a ser sujetos claves para un óptimo desempeño, donde no es menos importante la formación gerencial, concebida como el fruto de las experiencias y vivencias 
del gestor" (pág. 32). Es decir, se deben visualizar las capacidades y preparaciones requeridas para desempeñar su rol, a la vez de aflorar sus características personales.

\subsection{Perfil del Gerente del Siglo XXI}

Para que la gerencia del siglo XXI sea altamente eficaz, el perfil debe poseer ciertas características inherentes al cargo desempeñado según Aponte, (2012) como son: 1) Conocimiento del área específica; en vano es que el gerente desconozca características del área que supone gerenciar, por tanto se requiere de tiempo suficiente en la gestión para conocer de cerca los aspectos inherentes al cargo a desempeñar; 2) Visión de futuro, o sea capacidad de prever; en este momento gerente sin visión futurista no genera cambios o transformaciones en la empresa a regentar debe visualizar lo que se avizora a futuro para comenzar a cimentar las bases en la optimización de los retos planteados a posterior.

Dentro de este orden de ideas, 3) Capacidad de proveer información del mercado; para esto el gerente debe tener un conocimiento adecuado de los modelos de negocio, tanto de las empresas como de los pequeños comercios, que actualmente circulan en el mercado competitivo, aunado a sentir pasión por el negocio del que forma parte; 4) Teoría de las capacidades; se puede entender la necesidad que prevalece de tomar en cuenta los factores significativos en el bienestar y calidad de vida de todos los integrantes de la empresa que se gerencia; es decir supone que hay esferas importantes de actividad de las personas, donde el bienestar y la calidad de vida no son ocupación directa de las entradas económicas.

Asimismo, 5) Trabajar en equipo para análisis de escenarios; esta característica es relevante sin el trabajo en equipo no hay organización que se precie de cumplir con sus metas organizacionales, los equipos debidamente organizados darán auge a la empresa y será más fácil cumplir los objetivos 
propuestos; 6) Toma de decisiones bajo su única responsabilidad; en este aspecto las decisiones tomadas por el estratega deben ser con un conocimiento acabado de cuáles son los cambios que afectan el entorno y a la organización en su conjunto, dirigidos a tener un mejor impacto en su ejercicio general y, por ende, en los resultados.

Además, 7) Utiliza la Comunicación como su principal herramienta gerencial; esto es esencial la comunicación en todos sus aspectos genera inquietudes en los subordinados, una buena comunicación gerencial lleva a la empresa a un status superior y en armonía con todos para obtener el bien común, por último 8) Es creativo e innovador, sabe cómo se motiva y utiliza el resultado creativo de su gente, en el siglo XXI o siglo de la Creatividad, ésta no debe escapar a todas las actividades, transformaciones, cambios que se generen en la empresa, la creatividad ha sido y es producto de "genios" que han enaltecido organizaciones hoy día ocupando sitiales de honor y envergadura.

Las características antes mencionadas, son el resultado de una personalidad bien definida en gerentes que deseen cambiar, transformar, las organizaciones que están a su cargo. El cambio de milenio es una buena oportunidad, para reflexionar sobre las habilidades características que el nuevo entorno empresarial demanda de los gerentes.

Al respecto, Aponte, (ob. cit), expone "dirigir empresarialmente es obtener los objetivos planteados, llevando el timón del buque de la empresa o negocio en las manos, por medio de un equipo de personas -empleados de la empresa- y con medios materiales de que se dispone" (pág. 33). Eso se llama en la empresa "gestionar" los recursos para alcanzar los fines. Pues bien, para ser un buen gerente hay que llevar o sentir dentro el deseo de querer dirigir al grupo, querer pilotar ese buque. 


\subsection{El Pensamiento Estratégico como Modelo Gerencial}

El Pensamiento Estratégico se podría entender según, Ohmae, (ob.cit) como "la mezcla de métodos analíticos y flexibilidad mental utilizados para obtener ventajas competitivas" (pág. 89) En otras palabras, es el nacimiento para la toma de decisiones estratégica, ya que garantiza la salud a largo plazo de la empresa. Al respecto, Morrisey, (1996), establece "un pensador estratégico eficaz, tiene la capacidad de conectar sus acciones diarias con los objetivos a largo plazo del negocio. Esto significa tomar decisiones congruentes con las estrategias de la organización comprender cuando las propias estrategias necesitan evolucionar..." (pág. 31)

De hecho, el pensamiento estratégico provee las plataformas para la planeación estratégica con naturaleza intuitiva; lleva la figura, mientras que la planeación a largo alcance lleva a la visión y la planeación táctica al beneficio, a juicio de Morrisey, (ob. cit) "trata los valores, las convicciones filosóficas de los ejecutivos encargos de guiar a su empresa en un viraje exitoso, la misión y visión como conceptos actuales y futuros y la estrategia como la dirección en que debe avanzar la empresa" (pág. 11).

De este modo, desarrollar el pensamiento estratégico en una organización infiere la creación de un equipo directivo con una visión compartida de futuro y de un compromiso personal, una caracterizándose con el proyecto, estrategias y sentimiento profundo de autoridad, paternidad por parte de todas las personas claves de la organización. Cualquier cambio que se quiera introducir se pondrá en práctica mucho más fácil si se tiene el apoyo de todo el personal directivo de la empresa. En síntesis, antes de que la gerencia pueda decir que la empresa cuenta efectivamente con una nueva estrategia, es ineludible transformar con éxito las finalidades en realidades. 


\subsubsection{Los Modelos Gerenciales o Teorías Modernas}

Actualmente, las Teorías Gerenciales son estrategias de gestión organizacionales, utilizadas en la dirección, desarrollo del sistema y procesos de la misma. Todo modelo es una representación de una realidad que refleja, por lo que en gerencia, como en otras ciencias, los modelos o teorías determinaran una pauta, una base de sustento que a la larga permite el desarrollo orientado de la empresa u organización en general que lo utiliza. No obstante, ningún modelo por sí mismo basta para direccionar la empresa, por lo que su aplicación creativa es el secreto del éxito.

En este sentido, los modelos gerenciales se originan en las diferentes escuelas de pensamiento administrativo tanto clásicas como de última generación. Las escuelas de administración no necesariamente constituyen modelos de gerencia así como tampoco los modelos de Management (palabra inglesa para gerencia o administración que asume como sinónimos), se convierten en escuelas de pensamiento. Preferimos resolver este posible dilema, afirmando que los modelos gerenciales hacen parte de las estrategias que las empresas adoptan con el propósito de promover, mantener o impulsar su efectividad de gestión.

Referente a la teoría de la administración estratégica Robins y Coulter (2005) establecen "es utilizada por los gerentes para poder crear estrategias para la empresa, como planes de cómo la empresa llevará a cabo lo que requiera el negocio, como completará sus planes obtendrá clientes, para lograr el objetivo de estas estrategias" (pág. 182). La importancia que destacan de la administración estratégica, es que marca diferencias entre el desempeño de las diferentes empresas, ayuda a los gerentes a estar preparados a posibles cambios y ayuda a que los empleados se enfoquen, se esfuercen en lo que es más importante para la empresa.

Mientras tanto, Robbins y Coulter (ob. cit: 184) describen seis pasos en el proceso de la administración estratégica los cuales son: el primer paso 
consiste en identificar la misión actual de la empresa, objetivos y estrategias; el segundo realizar un análisis externo, el tercero hacer un análisis interno, el cuarto formular estrategias, quinto implementar estrategias y por ultimo evaluar las estrategias. El paso uno y dos es conocido como el análisis FODA en donde se analizan en la parte interna de la empresa las fortalezas y debilidades, las fortalezas son todas aquellas cosas que la empresa hace bien o recursos únicos que posee y las debilidades son las cosas que no hace bien la empresa o recursos que no posee. En la parte externa de la empresa se analizan las oportunidades y amenazas las oportunidades son todas aquellas tendencias positivas en el entorno externo y las amenazas son las tendencias negativas.

\subsubsection{Enfoques del Pensamiento Estratégico y su aplicación en las Organizaciones del Nuevo Milenio.}

Cuando se habla del pensamiento estratégico, se habla con relación a lo que puede ser una aproximación del mismo; no se trata necesariamente de un conjunto de teorías, se trata más bien, de los diferentes puntos de vista o enfoques que se han venido desarrollando. Asimismo, dentro del pensamiento estratégico, se encuentran enfoques definidos sobre la base de la forma en cómo se realiza el pensar estratégico y de cómo se establecen los objetivos, o se formula y ejecuta el plan. A continuación, se presentan los diversos enfoques del pensamiento estratégico.

A.- Enfoque Estrategia Versus Recursos: un primer enfoque apunta a que el pensamiento estratégico puede verse de dos modos: según Cendros, (2001) uno en el cual se establecen, en primer lugar, la visión, la misión y los objetivos; desarrollándose luego el plan estratégico en un contexto enunciativo de la visión que tiene la organización o el individuo del alcance de sus operaciones y acciones necesarias para lograr el éxito dentro de un ámbito particularmente competitivo. 
B.- Enfoque Central Versus Descentralizado: dependiendo de quién o quiénes realicen la planificación y del nivel de participación y decisión de los miembros de la organización, se consideran dos enfoques: El enfoque centralizado: la planificación se realiza a partir de un grupo selecto de individuos con capacidad para formular la misión, visión, objetivos, planes, recursos y el enfoque descentralizado: la organización formula sus planes en diferentes niveles, sobre la base de una misión y visión del negocio, las diferentes unidades o gerencias toman sus decisiones.

C.- La Planificación Participativa: En el enfoque participativo, se procura que la organización, en todos sus niveles, participe y haga aportes a la definición de la misión, visión, objetivos y metas que permitan un compromiso para garantizar el cumplimiento del plan estratégico diseñado, a favor de la organización que se regenta.

\section{Metodología}

La metodología es concebida como el estudio del método. Por esto, en esta indagación se asume la postura de Sautu, (2005):

La metodología, en tanto lógica de la investigación, se apoya en los paradigmas, su función investigativa es discutir fundamentos epistémicos del conocimiento...los métodos o modos de procedimiento son una serie de pasos que el investigador sigue en el proceso de producir una contribución al conocimiento (pág. 30).

En orden a estas premisas, el recorrido metodológico es la vía para llegar al camino que se desea indagar, por tanto el estudio se corresponde con una investigación de tipo documental, a juicio de Ortiz y García, (2002), "la investigación documental es la presentación de un escrito formal que sigue una metodología reconocida consiste primordialmente, en la presentación selectiva de lo que expertos ya han dicho o escrito sobre un tema determinado" (pág. 56). Por ende, la cuantía de indagación que se genera en todo el mundo 
es inmensa. Así, el adquirir estos conocimientos se hace con frecuencia mediante la investigación documental realizada en fuentes secundarias. Además, puede mostrar la posible unión de ideas entre varios autores y las del investigador. Su disposición demanda que éste reúna, interprete, evalúe e informe datos e ideas en forma ecuánime, decorosa y clara.

\section{Consideraciones Finales}

Los gerentes del siglo XXI serán similares a los de la década de los noventa, pero no iguales. La preparación será diferente y las formas de trabajo variarán. Idiomas, estudios, conocimientos informáticos y capacidad de comunicación son algunos de los aspectos a tener en cuenta para ser un gerente. Lo primero es tener ganas de conseguir el cargo; después, mucho sacrificio. La mejor medicina es la confianza y la peor la infravaloración personal o el desánimo.

Las características que hoy conocemos son útiles, pero cada vez se tornarán más inseparables; deberá ser estratega, pero al tiempo organizador y líder, pero para poder organizar necesita saber hacia dónde va, como va a organizarse, y en cada etapa saber ser líder, según se lo vaya exigiendo cada época de la historia, aunque a lo largo de la existencia del hombre en la tierra el líder siempre ha sido característico sobre los demás.

Deberá pues saber de todo lo bueno un poco, pero igualmente conocer de las cosas malas que pueden impresionar una organización, y ser consciente de que a medida que adelanta el tiempo además, de presentársele en el camino herramientas útiles para sobrellevar cualquier adversidad, aparecen también puntos negros que opacan el panorama. Hay que saber combinar en la proporción perfecta, habilidades técnicas, personales, específicas, y generales; "la idea es idear una máquina perfecta para la orientación con todos los conocimientos en cuanto a la administración se refiere, pero con mucho de corazón, y carisma entre las personas". 
En cuanto, al Pensamiento Estratégico. La dinámica competitiva de las organizaciones y de los mercados manifiesta un proceder según el cual, las que mejor se adecuen al entorno serán las que permanecerán en el tiempo. Esto debido, entre otras cosas, a que son las que están mejor equipadas y preparadas para enfrentar un ambiente de constante permutación e inseguridad. Por esto, el cambio permanente de las organizaciones obliga al desarrollo de una visión estratégica que permitan dar respuesta a las presiones del entorno y posicionar a la empresa competitivamente en el mercado.

Con base a lo expuesto, la estrategia a desarrollar debe concebirse como la búsqueda de un plan encaminado a desplazar ventajas competitivas que le permitan distinguirse de sus competidores, aprovechando sus fortalezas con el fin para los clientes. El enfoque estratégico implica la permanente búsqueda de la ventaja competitiva de crear un mayor valor agregado. Por lo tanto, el plan estratégico debe ser un instrumento flexible que evoluciona a la par de la empresa, mercados y clientes. Para ello es necesario considerar los tres elementos claves en torno al cual gira el análisis estratégico: empresa, clientes, y competencia.

\section{Referencias}

Añez, S. (2005). Pensamiento Estratégico en la Formación Gerencial del Alto Gerente Municipal. Revista Cuestiones Políticas Nº 34.

Cendros, J. (2001). Pensamiento Estratégico. Ensayos. Colección Gerencia de la Universidad Rafael Belloso Chacín. Ediciones Gato Azul. Venezuela.

Hernández, R. (2002). Determinación del Gerente de Recursos Humanos de las Empresas que le presta servicios a la Industria Petrolera. Formación Gerencial, Año 1, № 1, LUZ. Núcleo Costa Oriental del Lago. Venezuela. 
Morrisey, G. (1996). Pensamiento Estratégico. Construya los cimientos de su planeación. Ciudad de México: Editorial Prentice Hall Hispanoamericana.

Ohmahe, K. (2004). La mente del estratega. España: Editorial McGraw-Hill. Interamericana.

Sisk, H. y Sverdlik, M. (1979). Administración y Gerencia de Empresas. South-Westewrn Publishing Co. U.S.A 1979, 638 págs.

Sautu, R. (2005). Todo es teoría: objetivos y métodos de investigación. ed. Buenos Aires: Lumiere.

Ortiz, F. y García, M. (2002). Metodología de la Investigación: el Proceso y sus Técnicas. Ed. Limusa. 


\section{María Mercedes Carrillo}

e-mail: mercecar-42-68@hotmail.com

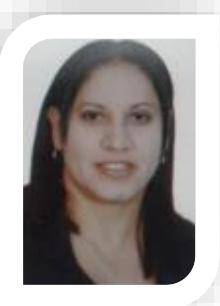

Nacida en Monte Carmelo, Estado Trujillo. Actualmente, cursa estudios de Doctorado en Ciencias de la Educación en la Universidad Nacional Experimental "Rafael María Baralt". Magister Sciantuarum en Administración de la Educación Básica. Licenciada en Educación Integral. Participante en el año 2010 de Actualización del Docente (300 horas). Ponente y participante del 1er y 2do Congreso Pedagógico en la Escuela Bolivariana "Antonio Nicolás Briceño", municipio Motatán, estado Trujillo. Experiencia Laboral actualmente docente de aula en la EPB "Antonio Nicolás Briceño". 